\title{
Andreev reflection through Fano resonances in molecular wires
}

\author{
A. Kormányos, I. Grace, and C. J. Lambert \\ Department of Physics, Lancaster University, Lancaster, LA1 4YB, UK
}

\begin{abstract}
We study Andreev reflection in a normal conductor-molecule-superconductor junction using a first principles approach. In particular, we focus on a family of molecules consisting of a molecular backbone and a weakly coupled side group. We show that the presence of the side group can lead to a Fano resonance in the Andreev reflection. We use a simple theoretical model to explain the results of the numerical calculations and to make predictions about the possible sub-gap resonance structures in the Andreev reflection coefficient.
\end{abstract}

PACS numbers: 73.63.-b,74.45.+c

Fano resonances $1 \frac{1}{=}$ are a universal interference phenomenon which can affect coherent electrical transport through nanostructures in many different systems. Examples of Fano lineshape in mesoscopic systems include scanning tunnelling microscope measurements on a single magnetic atom absorbed on a gold surface $^{2,3}$, single-electron transistors fabricated into a gated two-dimensional electron gas ${ }^{4}$, quantum dots embedded into an Aharonov-Bohm ring ${ }^{5,6}$, multiwall carbon nanotubes $\$ .8 .9$ and recently single-wall carbon nanotubes $\frac{10}{10}$ and double-wall nanotubes ${ }^{11}$. Fano resonances (FRs) also appear in the the conductance of quasi one-dimensional quantum wires with donor impurities 12 and in the case of quantum wires with a side coupled quantum $\operatorname{dot}^{13}$. In molecular electronics, due to the realistic treatment of the metal electrodes, FRs have been found in the transmission of dithiol benzene ${ }^{14}$. More generally, theoretical calculations predict that Fanolineshape should appear in the transmission through molecular wires with attached side groups $\frac{15}{5}$ or as a consequence of quantum interference between surface states of the measuring electrodes and the molecular orbitals 16 .

If one of the measuring probes is superconducting, the conductance for energies $E$ smaller than the superconducting pair potential $\Delta$ depends on the Andreev reflection probability $R_{A}(E)$. The Andreev reflection in various mesoscopic systems has been studied for a long time (see e.g Refs. 17,18 and references therein) but the interest has recently renewed when Andreev reflection through carbon nanotubes was measured experimentally 19,20 . These experiments have sparked numerous theoretical studies both in the absence of the electron-electron interaction 21,22 and in the presence of the interaction $23,24,25,26,27,28,29,30,31,32$. In many of these studies it was assumed that it was sufficient to consider resonant transport through a single energy level and as a consequence, the Andreev reflection as a function of energy exhibited Breit-Wigner type resonances. A notable exception is Ref. 33 where transport through an Aharonov-Bohm ring with an interacting quantum dot situated in one of its arms was considered and a Fano-type asymmetric resonance was found in the conductance. Very recently, Tanaka et al $\underline{34}^{34}$ studied Andreev transport through side-coupled interacting quantum dots focusing on the interplay of Andreev scattering and Kondo effect.

It was demonstrated in Ref. 15 that Fano resonances are a generic feature of molecular wires with attached side groups. It was also shown that for a certain type of molecular wires a FR can appear in the normal conductance $G_{N}(E)$ very close to the Fermi energy $E_{F}$. In a normal metal-molecule-superconductor (N-Mol-S) junction therefore these FRs would also affect the sub-gap transport. The aim of this paper is to study Andreev reflection through molecular wires when the normal conductance exhibits FRs close to the Fermi energy. Performing ab initio simulations of molecular wires in $\mathrm{N}$ Mol-S junctions we show how FRs influence the sub-gap transport. We elucidate the results of the numerical calculations using a simple analytic model. We also predict that for finite energies the differential conductance can reach the unitary limit if there is a strong asymmetry in the coupling to the leads.

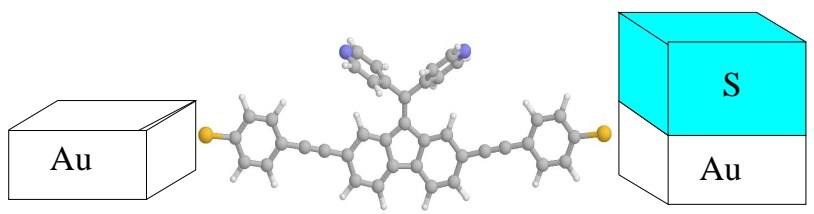

FIG. 1: Possible experimental setup. The molecule is contacted by gold electrodes, one of which is superconducting due to the proximity effect.

A possible experimental setup to measure Andreev reflection in N-Mol-S junctions is shown in Fig. 1. The molecule is contacted with thin gold electrodes on both sides. On top of one of the electrodes a second layer of e.g. aluminium or niobium is deposited, which at low enough temperature becomes superconducting. Due to the proximity effect this top layer induces superconductivity in the gold electrode beneath (in our calculations we assume that the induced superconductivity is $s$ type). We note that this setup was successfully used in Ref. 20 to study Andreev-reflecion in normal conductor - carbon nanotube - superconductor (N-Cn-S) junctions.

To study FRs in a N-Mol-S system, we choose the smallest molecule of a recently synthesized family of 
molecular wires $35,36,37$. Since these molecules have terminal thiol groups they can easily bind to gold surfaces making them ideal for experiments on single-molecule transport properties. The central part of the molecule consists of a single fluorenone unit, which could be chemically modified, e.g. by replacing the oxygen with bipyridine rings, as shown schematically in Fig. 11 The differential conductance of the system was calculated using a combination of the DFT code SIESTA 38 and a Green's function scattering approach explained in Refs. 39.40. Initially the isolated molecule is relaxed to find the optimum geometry, then the molecule is extended to include surface layers of the gold leads. In this way, charge transfer at the gold-molecule interface is included selfconsistently. The number $N_{g}$ of gold layers is increased until computed transport properties between the (normal conducting) gold leads no longer changed with increasing $N_{g}$. Typically, this extended molecule contained $N_{g}=3$ to 4 gold layers on each side, and the layers consisted of 9 atoms on the (111) plane. The leads, which were assumed to be periodic in the transport direction, also consisted of gold layers containing 9 atoms on the (111) plain. Using a double $-\zeta$ basis plus polarization orbitals, Troullier-Martins pseudopotentials $\underline{41}$ and the Ceperley-Alder LDA method to describe the exchange correlation ${ }^{42}$, effective tight-binding Hamiltonians $H_{M}$, $H_{L}$ of the extended molecule and of the leads, respectively, were obtained. To investigate the generic physics of this system, we employ the simplest possible approximation for the order parameter, namely that it changes in a step-function-like manner at the superconducting lead - extended molecule interface. Therefore the superconducting lead was modelled by introducing couplings of constant magnitude $\Delta$ between the electron and hole degrees of freedom in $H_{L}$, while no such coupling was present in the extended molecule and in the normal lead. We focus on sub-gap transport and therefore compute the Andreev-reflection probability $R_{A}(E)$, because at zero temperature for $E<\Delta$ the differential conductance is given by $G_{N S}(E)=\frac{4 e^{2}}{h} R_{A}(E)$.

The calculations of Ref. 15 have shown that by changing by rotational conformation of the bipyridine unit it is possible to change the position of the Fano resonance with respect to $E_{F}$. The definition of the angle of rotation $\theta$ of the bipyridine group is the following: $\theta=0^{\circ}$ when the rings of the sidegroup are parallel to the molecule axis and it is $90^{\circ}$ when they lie perpendicular.

We consider the molecule whose rotational conformation is $\theta=71.4^{\circ}$. Assuming first that both leads are normal conducting (N-Mol-N junction), close to the Fermi energy there is a FR in the differential conductance $G_{N}(E)=\frac{2 e^{2}}{h} T_{N}(E)$ (where $T_{N}(E)$ is the normal transmission) as it can be seen in Fig. 2. Since for conventional supercondcutors the typical superconducting gap values are $0.1-1.5 \mathrm{meV}$, we first consider the transport for energies $E<\Delta=1.35 \mathrm{meV}$. The Fano peak in $G_{N}(E)$ is at $\delta E \approx 3.7 \mathrm{meV}$ above $E_{F}$, therefore $\delta E$ is bigger than $\Delta$ and the influence of this resonance on the sub-gap transport can be understood by considering the zero bias conductance $G_{N S}(0)$. Indeed, as Fig. 3 shows, the Andreev reflection is almost constant apart from the region $E \approx \Delta$ where a sharp peak can be observed which is due to the singularity in the density of states of the superconductor at this energy. One can see that off-resonance

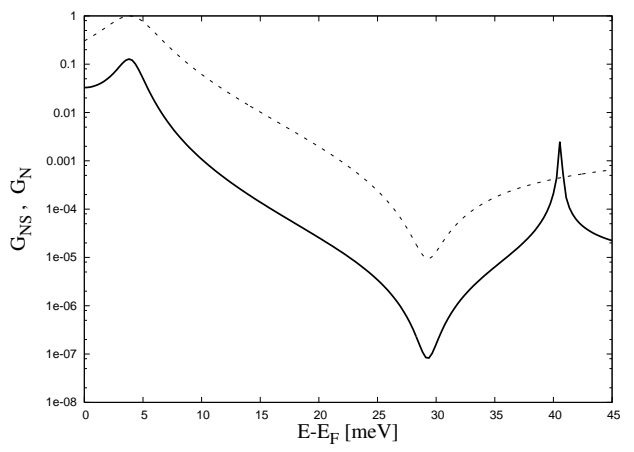

FIG. 2: Normal differential conductance $G_{N}(E)$ (in units of $2 e^{2} / h$, dashed line) and sub-gap conductance $G_{N S}(E)$ (in units of $4 e^{2} / h$, solid line) in logarithmic scale as a function of energy. We used $\Delta=41 \mathrm{meV}$.

$G_{N S}(0)$ is smaller than $G_{N}(0)$.

More generally however, if there is a narrow $\Gamma \lesssim \Delta$ resonance at some $|\delta E|<\Delta$ above or below $E_{F}$, the energy dependence of the Andreev reflection becomes important. (In case e.g. of carbon nanotubes, which can be gated, this scenario should be easily attainable, as in Ref 10 where the width of the Fano peak was $\approx 0.2 \mathrm{meV}$.) To illustrate this case, we performed computations using the same molecule but much bigger $\Delta$. The results of

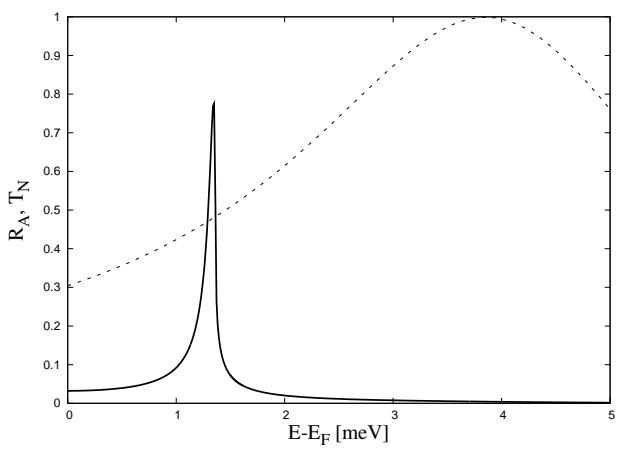

FIG. 3: $T_{N}(E)$ (dashed line) and $R_{A}(E)$ (solid line) as a function of energy. We used $\Delta=1.35 \mathrm{meV}$. The sharp peak in $R_{A}(E)$ is at $E \approx \Delta$ (see main text).

the computations are shown in Fig. 2, As one can see, a Fano resonance now appears both $G_{N}$ in $G_{N S}$. However, a closer inspection reveals that the width of the Fano peak in $G_{N S}$ at $\approx 3.7 \mathrm{meV}$ is roughly half of the width of the corresponding peak in $G_{N}$.

To explain the results of the numerical calculations we consider a simple model, introduced in Ref. 15, which was shown to capture the essential features of the transport between normal conducting leads. Close to a resonance, 
it is sufficient to consider a single backbone state $\left|f_{1}\right\rangle$ with resonant energy $\tilde{\varepsilon}_{1}$ and a state $\left|f_{2}\right\rangle$ of energy $\tilde{\varepsilon}_{2}$ which is associated with a side group of the molecule $\left(\tilde{\varepsilon}_{1}\right.$ and $\tilde{\varepsilon}_{2}$ are measured relative to the $E_{F}$ ). The weak coupling between the backbone of the molecule and the side group is described by a matrix $H_{12}$. We denote by $t_{c}=\left\langle f_{2}\left|H_{12}\right| f_{1}\right\rangle$ the coupling between the two states, whereas the coupling of the backbone state to the normal (superconductor) lead is described by matrices $W_{N}\left(W_{S}\right)$. A brief derivation of the Andreev reflection probability $R_{A}(E)$ for this system is given in Appendix $\mathrm{A}$, here we only summarize the main results.

The linear conductance is given by

$$
G_{N S}(0)=\frac{4 e^{2}}{h} \frac{4 \Gamma_{L}^{2} \Gamma_{R}^{2} \tilde{\varepsilon}_{2}^{4}}{\left[\left(\tilde{\varepsilon}_{+} \tilde{\varepsilon}_{-}\right)^{2}+\left(\Gamma_{L}^{2}+\Gamma_{R}^{2}\right) \tilde{\varepsilon}_{2}^{2}\right]^{2}} .
$$

Here $\Gamma_{L},\left(\Gamma_{R}\right)$ is the normal state tunnelling rate to the left (right) lead at $E_{F}$ and $\tilde{\varepsilon}_{ \pm}=\bar{\varepsilon} \pm \sqrt{\delta \varepsilon^{2}+t^{2}}$ where $\bar{\varepsilon}=$ $\left(\tilde{\varepsilon}_{1}+\tilde{\varepsilon}_{2}\right) / 2, \delta \varepsilon=\left(\tilde{\varepsilon}_{1}-\tilde{\varepsilon}_{2}\right) / 2$. The maximal conductance is attained at $\Gamma_{L}=\Gamma_{R}, \tilde{\varepsilon}_{ \pm}=0$ when it is twice as large as the normal conductance. Note that the conductance maximum is not attained when $\tilde{\varepsilon}_{1}$ is aligned with $E_{F}$ as one might expect. The hybridization between $\tilde{\varepsilon}_{1}$ and $\tilde{\varepsilon}_{2}$ due to the coupling $t_{c}$ leads to a different resonance condition for this system. Off-resonance, i.e. when $\tilde{\varepsilon}_{ \pm} \neq$ $0, G_{N S}$ falls off more rapidly as a function of $\tilde{\varepsilon}_{ \pm}$than $G_{N}$ [see Eq. (1) in Ref. 15]. Therefore the $G_{N S}(0)$ is usually smaller than $G_{N}(0)$. Moreover, $G_{N S}$ is zero if $\tilde{\varepsilon}_{2}=0$, i.e. when the energy of the side coupled state equals $E_{F}$.
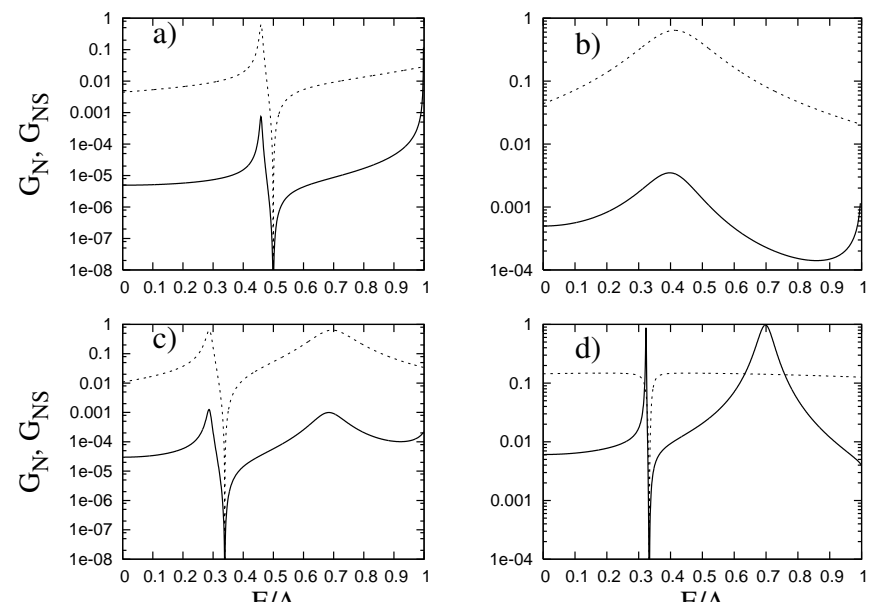

FIG. 4: $G_{N}$ (in units of $2 e^{2} / h$, dashed) and $G_{N S}$ (in units of $4 e^{2} / h$, solid) in logarithmic scale as a function of energy. We used $\sqrt{\Gamma_{N}^{e} \Gamma_{N}^{h}} /\left|\sigma_{S}^{e h}\right|=4$ in the case of a), b), c) and $\sqrt{\Gamma_{N}^{e} \Gamma_{N}^{h}} /\left|\sigma_{S}^{e h}\right|=0.25$, in the case of d).

For finite energies $E<\Delta$ the most important features of the differential conductance of our model are the following. If the coupling to the normal lead is stronger than to the superconducting one, i.e. when $\sqrt{\Gamma_{N}^{e} \Gamma_{N}^{h}} \gtrsim\left|\sigma_{S}^{e h}\right|$ where $\Gamma_{N}^{e}\left(\Gamma_{N}^{h}\right)$ are tunnelling rates for electrons (holes) from the normal lead and $\sigma_{S}^{e h}$ is an off-diagonal element of the self-energy matrix $\Sigma_{S}$ (see Appendix $\AA$ for the precise definitions, as well as for the definitions of $\varepsilon_{+}^{e}, \varepsilon_{-}^{e}$, to be introduced below), in good approximation

$$
G_{N S}(E)=\frac{4 e^{2}}{h} A(E) T_{e}(E)
$$

where the amplitude $A(E)$ is a slowly varying function of the energy and

$$
T_{e}(E)=\frac{\Gamma_{N}^{e} \Gamma_{N}^{h}\left(E-\tilde{\varepsilon}_{2}\right)^{2}}{\left[\left(E-\varepsilon_{+}^{e}\right)\left(E-\varepsilon_{-}^{e}\right)\right]^{2}+\left(\Gamma_{N}^{e}\right)^{2}\left(E-\tilde{\varepsilon}_{2}\right)^{2}} .
$$

Assuming a weak coupling between $\left|f_{1}\right\rangle$ and $\left|f_{2}\right\rangle$ i.e. that $t_{c} \ll \delta \varepsilon=\left|\varepsilon_{+}^{e}-\varepsilon_{-}^{e}\right|$ (which also means that $\varepsilon_{-}^{e} \approx \tilde{\varepsilon}_{2}$ ), for energies close to $\varepsilon_{-}^{e}$ the probability amplitude $T_{e}(E)$ can be further approximated by

$$
T_{e}(E) \approx \mathcal{A} \frac{(\epsilon+q)^{2}}{\alpha^{2} \epsilon^{2}+1}
$$

where $\mathcal{A}=\Gamma_{N}^{e} \Gamma_{N}^{h} /\left(\varepsilon_{-}^{e}-\tilde{\varepsilon}_{2}\right)^{2}, \epsilon=\left(E-\varepsilon_{-}^{e}\right) / \Gamma_{N}^{e}, \alpha^{2}=$ $\left(\varepsilon_{-}^{e}-\varepsilon_{+}^{e}\right)^{2} /\left(\varepsilon_{-}^{e}-\tilde{\varepsilon}_{2}\right)^{2}$ and $q=\left(\varepsilon_{-}^{e}-\tilde{\varepsilon}_{2}\right) / \Gamma_{N}^{e}$. Therefore, if $0<\varepsilon_{-}^{e}, \tilde{\varepsilon}_{2}<\Delta$ a FR will appear in the subgap transport [see Fig 4(a)]. For strong coupling such that $\Gamma_{N}^{e} \gg\left|\varepsilon_{-}^{e}-\tilde{\varepsilon}_{2}\right|$ the Fano lineshape would become a symmetric dip. If however $\varepsilon_{+}^{e}<\Delta<\varepsilon_{-}^{e}, \tilde{\varepsilon}_{2}$ is satisfied, a Breit-Wigner resonance (BWR) of width $\Gamma_{N}^{e}$ occurs [shown in Fig. 4(b)], while for $0<\varepsilon_{+}^{e}, \varepsilon_{-}^{e}, \tilde{\varepsilon}_{2}<\Delta$ the Andreev reflection exhibits both a FR and a BWR [Fig. 4(c)]. Note, that $T_{e}(E)$ is very similar to the transmission amplitude $T_{N}(E)$ calculated in Ref. 15 for normal conducting leads. Since for $\sqrt{\Gamma_{N}^{e} \Gamma_{N}^{h}} \gtrsim\left|\sigma_{S}^{e h}\right|$ the resonance energies $\varepsilon_{-}^{e}, \varepsilon_{+}^{e}$ are usually very close to the resonance energies appearing in the expression of $T_{N}(E)$, one finds that the resonance structures of the normal conductance will also appear in the sub-gap transport if the relevant resonance energies are smaller than the superconducting pair potential. This explains the occurrence of a Fano resonance in $G_{N S}(E)$ in Fig. 2, However, since $A(E)$ in Eq. (2D) is usually much smaller than unity, $G_{N S}(E)$ itself can also be smaller than $G_{N}(E)$. The widths of the resonances in the Andreev-reflection coefficient can be significantly smaller than in the normal transmission. This happens because coupling to the superconductor does not lead to the broadening of the resonant levels. Therefore if $\sqrt{\Gamma_{N}^{e} \Gamma_{N}^{h}} \gg\left|\sigma_{S}^{e h}\right|$ the peaks in the normal and in the Andreev transport have roughly the same width while for $\sqrt{\Gamma_{N}^{e} \Gamma_{N}^{h}} \gtrsim\left|\sigma_{S}^{e h}\right|$ the width of the peaks in the Andreev reflection is half of the width of the corresponding peaks in the normal transmission. This can also be observed in Fig. 2. We note that for $E \approx \Delta$ where $\sigma_{S}^{e h}$ changes rapidly with energy the formula shown in Eq. (2) is not applicable because in the derivation of Eq. (2) we have assumed that the self energy $\sigma_{S}^{e h}$ is a slowly varying function of the energy. 
Finally, we briefly discuss the predictions of our model for the case when the coupling to the superconductor is stronger than to the normal lead, i.e. when $\left|\sigma_{S}^{e h}\right| \gtrsim$ $\sqrt{\Gamma_{N}^{e} \Gamma_{N}^{h}}$. The conductance can no longer be approximated by Eq. (2) because $\sigma_{S}^{e h}$ introduces hybridization between electron and hole levels. We find that in the most general case the conductance exhibits both a FR and a BWR, if the corresponding resonance energies are smaller than the superconducting gap. These peaks, as mentioned before, can be much narrower than the ones in the normal transmission because the superconductor does not broaden them. Moreover, we find that for $\left|\sigma_{S}^{e h}\right| \gg \sqrt{\Gamma_{N}^{e} \Gamma_{N}^{h}}$ the conductance can even reach the unitarity limit. This could not happen in the opposite, $\left|\sigma_{S}^{e h}\right| \ll \sqrt{\Gamma_{N}^{e} \Gamma_{N}^{h}}$ case because a resonance in $T_{e}(E)$ is not accompanied by a resonance in $A(E)$ and therefore the conductance is always smaller than $4 e^{2} / h$. We illustrate this in Fig. 4(d) where $G_{N S}$ is shown along with $G_{N}$. One can see that $G_{N}<2 e^{2} / h$ because the couplings to the leads are asymmetric and there is a broad resonance at $E / \Delta \approx 0.15$ along with an almost symmetric, narrow dip at $E / \Delta \approx 0.32$. In contrast, $G_{N S}$ has a narrow FR and also a BWR, the latter peak reaching the unitarity limit.

In summary, we have studied the Andreev reflection through a class of molecules which exhibit Fano resonances in the normal conductance. Our numerical calculations based on $a b$ initio methods indicate that Fano resonances may also appear in the sub-gap transport. A simple theoretical model that we used to understand the results of the numerical calculations predicts that a) if the coupling to the normal lead is weaker than the coupling to the superconducting one, the resonance structure of the normal conductance can manifest itself in the Andreev reflection coefficient if the resonance energies are smaller than the superconducting gap and b) if the coupling to the superconductor is strong, the resonances in the normal conductance and in the Andreev reflection can be very different, both in position and in width.

\section{ACKNOWLEDGMENT}

This work is supported partly by European Commission Contract No. MRTN-CT-2003-504574 and by EPSRC.

\section{APPENDIX A}

There are numerous equivalent approaches to calculate transport coefficients through phase coherent normal- superconductor hybrid systems ${ }^{43}$. Here we employ the Green's function technique presented in Ref. 44 in which the Hilbert space is divided into a sub-space $A$ containing the external leads and a sub-space $B$ containing the molecule.

Assuming for a moment that the molecule is isolated, for energies close to a resonance it can be described by quantum states $\left|f_{1}\right\rangle,\left|f_{2}\right\rangle$ with resonant energies $\varepsilon_{1}, \varepsilon_{2}$. These states are coupled together by a hamiltonian $H_{12}$ with matrix element $t_{c}=\left\langle f_{1}\left|H_{12}\right| f_{2}\right\rangle$. The effect of coupling of the molecule to the normal conducting (superconducting) lead via a coupling matrix $W_{N}\left(W_{S}\right)$ is represented by the energy dependent self-energy matrices $\boldsymbol{\Sigma}_{N}=\boldsymbol{\sigma}_{N}-i \boldsymbol{\Gamma}_{N}\left(\boldsymbol{\Sigma}_{S}=\boldsymbol{\sigma}_{S}-i \boldsymbol{\Gamma}_{S}\right)$ where $\boldsymbol{\sigma}_{N}, \boldsymbol{\Gamma}_{N}\left(\boldsymbol{\sigma}_{S}\right.$, $\left.\boldsymbol{\Gamma}_{S}\right)$ are hermitian. We assume that the coupling matrices are diagonal in the quasiparticle $e, h$ space:

$$
W_{N, S}=\left(\begin{array}{cc}
W_{N, S}^{e} & 0 \\
0 & W_{N, S}^{h}
\end{array}\right)
$$

where $W_{N(S)}^{e}=-\left(W_{N(S)}^{h}\right)^{*}$. Since the Green's function of the (isolated) normal lead is also diagonal in the quasiparticle space, so will be $\boldsymbol{\Sigma}_{N}=\operatorname{Diag}\left(\boldsymbol{\sigma}_{N}^{e}-i \boldsymbol{\Gamma}_{N}^{e}, \boldsymbol{\sigma}_{N}^{h}-\right.$ $\left.i \boldsymbol{\Gamma}_{N}^{h}\right)$, too. The self energy coming from the coupling to the superconductor has both diagonal and off-diagonal parts, but for $E \leq \Delta$ it reads

$$
\boldsymbol{\Sigma}_{S}=\left(\begin{array}{cc}
\boldsymbol{\sigma}_{S}^{e} & \boldsymbol{\sigma}_{S}^{e h} \\
\boldsymbol{\sigma}_{S}^{h e} & \boldsymbol{\sigma}_{S}^{h}
\end{array}\right)
$$

i.e. the superconducting lead does not broaden the levels. Moreover, since $\left|f_{2}\right\rangle$ is only coupled with $\left|f_{1}\right\rangle$ but not with any of the leads, the self-energy matrix elements of the matrices $\boldsymbol{\Sigma}_{N}, \boldsymbol{\Sigma}_{S}$ will only affect the resonance energy $\varepsilon_{1}$ of the backbone state but not the energy $\tilde{\varepsilon}_{2}=\varepsilon_{2}-E_{F}$ of the side coupled state. We now introduce the following notations: $\tilde{\varepsilon}_{1}^{e, h}=\varepsilon_{1}-E_{F}-\left(\sigma_{N}^{e, h}+\sigma_{S}^{e, h}\right)$ [where $\tilde{\sigma}_{N, S}^{e, h}$ are the (only) nonzero element of the matrices $\left.\boldsymbol{\sigma}_{N, S}^{e, h}\right], \bar{\varepsilon}^{e, h}=\left(\tilde{\varepsilon}_{1}^{e, h}+\tilde{\varepsilon}_{2}\right) / 2, \delta \varepsilon^{e, h}=\left(\tilde{\varepsilon}_{1}^{e, h}-\tilde{\varepsilon}_{2}\right) / 2$, $\varepsilon_{ \pm}^{e, h}=\bar{\varepsilon}^{e, h} \pm \sqrt{\left(\delta \varepsilon^{e, h}\right)^{2}+t_{c}^{2}}$. Denoting by $\mathbf{G}_{B B}(E)$ the retarded Green's function of the molecule and using the formula 44

$$
R_{A}=\operatorname{Tr}\left[\boldsymbol{\Gamma}_{N}^{e} \mathbf{G}_{B B}(E) \boldsymbol{\Gamma}_{N}^{h} \mathbf{G}_{B B}^{\dagger}(E)\right]
$$

to calculate the probability of the Andreev reflection, we find after straightforward calculations that

$$
R_{A}=\frac{4 \Gamma_{N}^{e} \Gamma_{N}^{h}\left(E-\tilde{\varepsilon}_{2}\right)^{2}\left(E+\tilde{\varepsilon}_{2}\right)^{2}\left(\sigma_{S}^{e h}\right)^{2}}{|D|^{2}}
$$

Here the denominator is 


$$
D=\left[\left(E-\varepsilon_{+}^{e}\right)\left(E-\varepsilon_{-}^{e}\right)+i\left(E-\tilde{\varepsilon}_{2}\right) \Gamma_{N}^{e}\right]\left[\left(E+\varepsilon_{+}^{h}\right)\left(E+\varepsilon_{-}^{h}\right)+i\left(E+\tilde{\varepsilon}_{2}\right) \Gamma_{N}^{h}\right]-\left(\sigma_{S}^{e h}\right)^{2}\left(E-\tilde{\varepsilon}_{2}\right)\left(E+\tilde{\varepsilon}_{2}\right) .
$$

and $\sigma_{S}^{e h}, \Gamma_{N}^{e}, \Gamma_{N}^{h}$ are the only non-zero elements of the

matrices $\boldsymbol{\sigma}_{S}^{e h}=\boldsymbol{\sigma}_{S}^{h e}, \boldsymbol{\Gamma}_{N}^{e}, \boldsymbol{\Gamma}_{N}^{h}$.

1 U. Fano, Phys. Rev. 124, 1866 (1961).

${ }^{2}$ V. Madhavan, W. Chen, T. Jamneala, M. F. Crommie, and N. S. Wingreen, Science, 280, 567, (1998).

3 J. Li, W. D. Schneider, R. Berndt, and B. Delley, Phys. Rev. Lett. 80, 2893 (1998).

4 J. Göres, D. Goldhaber-Gordon, S. Heemeyer, M. A. Kastner, H. Shtrikman, D. Mahalu, and U. Meirav, Phys. Rev. B 62, 2188 (2000).

${ }^{5}$ K. Kobayashi, H. Aikawa, S. Katsumoto, and Y. Iye, Phys. Rev. Lett. 88, 256806 (2002).

${ }^{6}$ K. Kobayashi, H. Aikawa, S. Katsumoto, and Y. Iye, Phys. Rev. B 68, 235304 (2003).

7 J. Kim, J. R. Kim, Jeong-O Lee, J. W. Park, H. M. So, N. Kim, K. Kang, K. H. Yoo, and J. J. Kim, Phys. Rev. Lett. 90166403 (2003).

${ }^{8}$ W. Yi, L. Lu, H. Hu, Z. W. Pan, and S. S. Xie, Phys. Rev. Lett., 91, 076801, (2003).

9 Z. Zhang, V. Chandrasekhar, D. A. Dikin, and R. S. Ruoff, cond-mat/0311360v1, (2003).

10 B. Babić and C. Schönenberger, Phys. Rev. B 70, 195408 (2004).

11 I. M. Grace, S. W. Bailey, and C. J. Lambert, Phys. Rev. B 70, 153405 (2004).

12 E. Tekman and P.F. Bagwell, Phys. Rev. B 48, 2553 (1993).

13 R. Franco, M.S. Figueira and E.V. Anda, Phys. Rev. B 67, 155301 (2003).

14 A. Grigoriev, J. Sköldberg, G. Wendin, and Z. Crljen, Phys. Rev. B 74, 045401 (2006).

15 T. A. Papadopoulos, I. M. Grace, and C. J. Lambert, Phys. Rev. B 74, 193306 (2006).

16 Xingqiang Shi, Zhenxiang Dai, and Zhi Zeng, Phys. Rev. B 76, 235412 (2007).

17 C. W. J. Beenakker, Rev. Mod. Phys. 69731 (1997).

18 C. J. Lambert and R. Raimondi, J. Phys.:Condens. Matter 10901 (1998).

19 A. F. Morpurgo, J. Kong, C. M. Marcus, and H. Dai, Science 286263 (1999).

20 M. Gräber, T. Nussbaumer, W. Belzig and C. Schönenberger, Nanotechnology 15, S479 (2004).

21 Y. Wei, J. Wang, H. Guo, H. Mehrez, and Ch. Roland, Phys. Rev. B 63, 195412 (2001).

${ }^{22}$ Hui Pan, Tsun-Han Lin, and Dapeng Yu, Phys. Rev. B 70, 245412 (2004).

23 M. Titov, M. Múller, and W. Belzig, Phys. Rev. Lett. 97,
237006 (2006).

24 S. Vishveshwara, C. Bena, L. Balents, and Matthew P. A. Fisher, Phys. Rev. B 66, 165411 (2002).

25 J. C. Cuevas, A. Levy Yeyati, and A. Martn-Rodero, Phys. Rev. B 63, 094515 (2001).

26 P. Schwab and R. Raimondi, Phys. Rev. B 59, 1637 (1999).

27 A. A. Clerk, V. Ambegaokar, and S. Hershfield, Phys. Rev. B 61, 3555 (2000).

28 Q.-F. Sun, H. Guo, and T.-H. Lin, Phys. Rev. Lett. 87, 176601 (2001).

29 Y. Avishai, A. Golub, and A. D. Zaikin, Phys. Rev. B 63, 134515 (2001).

30 J. Splettstoesser, M. Governale, J. König, F. Taddei, and R. Fazio, Phys. Rev. B 75, 235302 (2007).

31 T. Domański, A. Donabidowicz, and K. I. Wysokiński, Phys. Rev. B 76, 104514 (2007).

32 T. Domański and A. Donabidowicz Phys. Rev. B 78, 073105 (2008).

33 Anatoly Golub and Yshai Avishai, Phys. Rev. B 69, 165325 (2004).

34 Y. Tanaka,N. Kawakami, and A. Oguri, Phys. Rev. B 78, 035444 (2008).

35 C. Wang, A. S. Batsanov, M. R. Bryce and I. Sage, Org. Lett, 6 (13), 2181 (2004).

36 C. Wang, A. S. Batsanov and M. R. Bryce, Faraday Discussions, 131, 221-234 (2006).

37 C. Wang, A. S. Batsanov and M. R. Bryce, J. Org. Chem. 71, 108-116 (2006).

38 J. M. Soler, E. Artacho, J. D. Gale, A. Garcia, J. Junquera, P.Ordejon and D. Sanchez-Portal, J.Phys.: Condens. Matter 14, 2745 (2002).

39 S. Sanvito, C. J. Lambert, J. H. Jefferson and A. M. Bratkovsky, Phys. Rev. B 59, 11936 (1999).

40 A. R. Rocha, V. M. García-Suárez, S. Bailey, C. J. Lambert, J. Ferrer, and S. Sanvito, Phys. Rev. B 73, 085414 (2006).

41 N. Troullier and Jos Luriaas Martins, Phys. Rev. B 43, 1993 (1991).

42 J. P. Perdew, A. Zunger, Phys. Rev. B 23, 5048 (1981).

43 C. J. Lambert, J. Phys. C 17, 2401 (1984), V. C. Hui, C. J. Lambert, Europhys. Lett. 23, 203 (1993). C.J. Lambert, J. Phys. C 17, 2401 (1984)

44 N. R. Claughton, M. Leadbeater and C. J. Lambert, J. Phys: Condens. Matter 78757 (1995). 